\title{
Sinulation of a Wavelength-modulation Lorentzian Lineshape Function
}

\author{
Jian Li, Ye Mu and Yue Qu \\ Information technology college of Jilin Agricultural University. Changchun 130118, China \\ 342884220@qq.com
}

\begin{abstract}
Keywords: Wavelength modulation; Fourier components; Lorentzian; MATLAB
\end{abstract}
\begin{abstract}
A theoretical description of the wavelength-modulation spectrometry Lorentzian lineshape function in terms of a normalized detuning and normalized modulation amplitude is given based on Fourier analysis. Simplified Lorentzian function lineshape expressions for the even harmonics of the Fourier components on resonance, given in terms of the normalized modulation amplitude, are simulation based on Matlab. The normalized modulation amplitudes that maximize the four lowest even harmonics of Fourier components are 2.20, 4.12, 6.08, and 8.06, respectively.
\end{abstract}

\section{Introduction}

Wavelength-modulation spectrometry (WMS) together with laser absorption is nowadays a useful technique for sensitive trace-species detection $1-4$. When these attributes are combined with various modulation techniques astonishing detection limits are achieved under a variety of conditions5-6. Modulation of the light from a diode laser can most conveniently be accomplished by modulation of the injection current, which imposes modulation on the frequency of the emitted light. One important advantage of modulation techniques is that they shift the detection to high frequencies to reduce $1 / \mathrm{f}$ noise, which decreases with increasing frequency.

A general formula for the nth Fourier component of a wavelength-modulated Lorentzian function has previously been given by Arndt[7]. That expression is, however, given as a sum of a term encompassing various combinations (sums, square roots, and powers) of complex expressions of the normalized detuning and modulation amplitude and its complex conjugate. This makes that particular expression far from transparent for analytical analyses and only useful when computer programs that can handle complex routines numerically can be used. We present therefore, in this paper, a simulation for the nth Fourier component of a wavelength-modulated Lorentzian lineshape function based on MATLAB.

\section{Modulation Theory}

In WMS, the wavelength is sinusoidally modulated through a corresponding modulation of the injection current. Applying a small sinusoidal modulation of frequency $f_{m}$ to the injection current of a laser results in a modulation of the laser frequency, $v(t)=v_{c}+v_{a} \cos \left(2 \pi f_{m} t\right)$

Where $v_{c}$ is a center laser frequency and $\bar{v}_{a}$ represents the frequency modulation amplitude. It is, however, often convenient to express the laser frequency entities normalized in terms of the position and half-width-half-maximum width (HWHM) of the absorption profile $\Delta v$.

$\bar{v}_{d}=\left(v_{c}-v_{0}\right) / \Delta v \quad \bar{v}_{a}=v_{a} / \Delta v$

where $\bar{v}_{d}$ is the detuning of the laser center frequency from the center frequency of the absorption line normalized with respect to the width of the absorption profile, $\bar{v}_{a}$ is the width-normalized frequency modulation amplitude, and $\Delta v$ is the half width at half-maximum (HWHM) of the Lorentzian lineshape function.Let us therefore write $\bar{v}(t)=\bar{v}_{d}+\bar{v}_{a} \cos (2 \pi f t)$ 
Kluczynski and Axner calculated therefore explicitly in their work expressions in real forms for the five first Fourier components $(\mathrm{n}=0-4)$ of a Lorentzian lineshape function ${ }^{[8]}$.

$$
\begin{aligned}
& S_{\mathrm{AS}, 2}^{\text {even }}\left(\bar{v}_{d}, \bar{v}_{a}\right)=-\beta \alpha_{0} I\left(v_{c}\right)\left[\bar{\chi}_{2}^{e} \bar{I}_{0}^{e}+0.5\left(\bar{\chi}_{1}^{e}+\bar{\chi}_{3}^{e}\right) \bar{I}_{1}^{e}+\left(\bar{\chi}_{0}^{e}+0.5 \bar{\chi}_{4}^{e}\right) \bar{I}_{2}^{e}+0.5\left(\bar{\chi}_{1}^{e}+\bar{\chi}_{5}^{e}\right) \bar{I}_{3}^{e}+0.5\left(\bar{\chi}_{2}^{e}+\bar{\chi}_{6}^{e}\right) \bar{I}_{4}^{e}+\ldots\right] \\
& S_{\mathrm{AS}, 4}^{\text {even }}\left(\bar{v}_{d}, \bar{v}_{a}\right)=-\beta \alpha_{0} I\left(v_{c}\right)\left[\bar{\chi}_{4}^{e} \bar{I}_{0}^{e}+0.5\left(\bar{\chi}_{3}^{e}+\bar{\chi}_{5}^{e}\right) \bar{I}_{1}^{e}+0.5\left(\bar{\chi}_{2}^{e}+\bar{\chi}_{6}^{e}\right) \bar{I}_{2}^{e}+0.5\left(\bar{\chi}_{1}^{e}+\bar{\chi}_{7}^{e}\right) \bar{I}_{3}^{e}+\left(\bar{\chi}_{0}^{e}+0.5 \bar{\chi}_{8}^{e}\right) \bar{I}_{4}^{e}+\ldots\right]
\end{aligned}
$$

Where $\beta$ is an instrument factor, $\alpha_{0}$ the optical thickness of the sample on resonance, $I(v)$ the intensity seen by the detector, $\bar{\chi}_{n}^{e}$ the nth (even) Fourier component of the peak-normalized lineshape function, and $\bar{I}_{n}^{e}$ the nth (even) normalized Fourier components of the intensity.

The absorption cross section consists of an intensity term and a line-shape function, which is approximated by a Lorentzian function in this work. can be written as An simply time-dependent analytical expression for a wavelength-modulated peak-normalized Lorentzian shaped lineshape function can then be written in terms of the normalized detuning, $\bar{v}_{d}$, and normalized modulation amplitude, $\bar{v}_{a}$, as $\bar{\chi}(t)=\frac{(\Delta v)^{2}}{\left\{v_{0}-\left[v_{c}+v_{a} \cos (2 \pi f t)\right]\right\}^{2}+(\Delta v)^{2}}=\frac{1}{1+\left[\bar{v}_{d}+\bar{v}_{a} \cos (2 \pi f \mathrm{t})\right]^{2}}=\frac{1}{1+\bar{v}^{2}(t)}(6)$

Arndt has previously derived an analytical expression for the nth harmonic of the wavelength modulated peak-normalized Lorentzian shaped lineshape function

$$
\bar{\chi}\left(\bar{v}_{d}, \bar{v}_{a}\right)=\frac{2-\delta_{n 0}}{2} \frac{\left\{\sqrt{\left[\left(1-\mathrm{i} \bar{v}_{d}\right)^{2}+\bar{v}_{a}^{2}\right]}-\left(1-\mathrm{i} \bar{v}_{d}\right)\right\}^{n}}{\bar{v}_{a}^{n} \sqrt{\left[\left(1-\mathrm{i} \bar{v}_{d}\right)^{2}+\bar{v}_{a}^{2}\right]}} \mathrm{i}^{n}+\text { c.c. }
$$

where c.c. stands for the complex conjugate. However, its form is rather complicated; wherefore it is difficult to use it for analytical purposes, even to the lowest orders.

Axner et al. derived a general, non-complex analytical expression for the even component of the nth Fourier coefficient of a wavelength-modulated Lorentzian lineshape function. An expression for the nth Fourier component of the Lorentzian lineshape function can therefore finally be written succinctly as

$$
\bar{\chi}\left(\bar{v}_{d}, \bar{v}_{a}\right)=\frac{A_{n}}{\bar{v}_{a}^{n}}\left[B_{n}+\frac{C_{n} S_{+}+D_{n} S_{-}}{\sqrt{2} \mathrm{R}}\right]
$$

$$
\begin{array}{ll}
\mathrm{M}=1+\bar{v}_{a}^{2}-\bar{v}_{d}^{2} & S_{+}=\sqrt{\mathrm{R}+\mathrm{M}} \\
\mathrm{R}=\sqrt{\mathrm{M}^{2}+4 \bar{v}_{d}^{2}} & S_{-}=\sqrt{\mathrm{R}-\mathrm{M}}
\end{array}
$$

The entities are furthermore solely functions of and.Graphical representation of the Fourier components of a Lorentzian function as a function of detuning

Using the description of the various Fourier components from above, it is possible to obtain fairly simple expressions for the even harmonics of the Fourier components on resonance as a function of normalized modulation amplitude. The general expression was shown, that the signal strength for the even harmonics of the Fourier components on resonance can be written in a particularly simple form, namely as

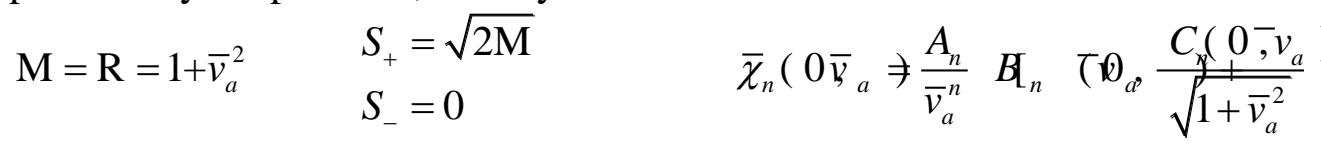

which gives rise to clearly manageable expressions for the $\bar{v}_{a}$ dependence of the various even nth Fourier components on resonance. The four lowest even harmonicsare given in Table 1.

Table 1 Explicit expressions for the four lowest even harmonics $(n=2,4,6$, and 8) of the Fourier components of a wavelength modulated Lorentzian function on resonance, $\bar{\chi}_{n}\left(0, \bar{v}_{a}\right)$

Component Expression 


$$
\begin{aligned}
& \bar{\chi}_{2}\left(0, \bar{v}_{a}\right) \quad-\frac{2}{\bar{v}_{a}^{2}}\left[2-\frac{2+\bar{v}_{a}^{2}}{\sqrt{1+\bar{v}_{a}^{2}}}\right. \\
& \bar{\chi}_{4}\left(0, \bar{v}_{a}\right) \\
& -\frac{2}{\bar{v}_{a}^{4}}\left[4\left(2+\bar{v}_{a}^{2}\right)-\frac{8+8 \bar{v}_{a}^{2}+\bar{v}_{a}^{4}}{\sqrt{1+\bar{v}_{a}^{2}}}\right] \\
& \bar{\chi}_{6}\left(0, \bar{v}_{a}\right) \\
& \frac{2}{\bar{v}_{a}^{6}}\left[2\left(6+16 \bar{v}_{a}^{2}+3 \bar{v}_{a}^{4}\right)-\frac{32+48 \bar{v}_{a}^{2}+18 \bar{v}_{a}^{4}+\bar{v}_{a}^{6}}{\sqrt{1+\bar{v}_{a}^{2}}}\right] \\
& \bar{\chi}_{8}\left(0, \bar{v}_{a}\right)
\end{aligned}
$$

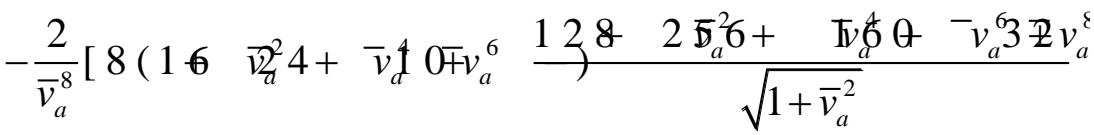

\section{Sinulation}

Fig. 1 shows the eighth lowest Fourier components (the odd harmonics in Fig. 1a, i.e. n=1, 3, 5, and 7, and the even in Fig. 1b, i.e. $\mathrm{n}=2,4,6$, and 8) of a wavelength-modulated Lorentzian function as a function of $\bar{v}_{d}$, for the $\bar{v}_{a}$ values that maximize each individual harmonic. It is interesting to note that while Fig. 1 shows the well-known fact that the even harmonics of the Fourier components take their maximum value at resonance and the odd components take their maximum value at a finite detuning, Fig.1a show that the optimum detuning for the odd components is almost independent of the order of the Fourier component.
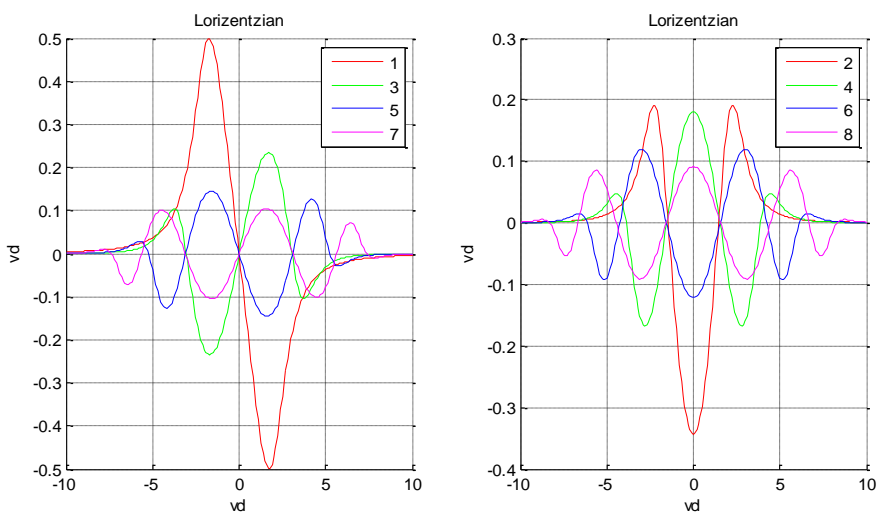

Figure 1. The four lowest harmonics of the Fourier components of Lorentzian function at detuning.

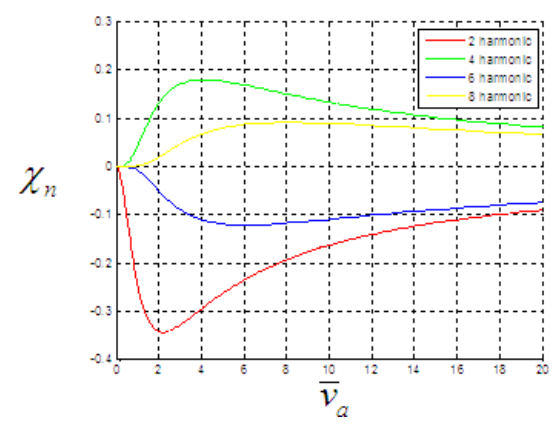

Figure 2. The four lowest even harmonics of the Fourier components of Lorentzian function on resonance as a function of normalized frequency modulation amplitude. 
Fig. 2 shows the four lowest even harmonics of the Fourier components of a wavelength modulated Lorentzian function on resonance as a function of modulation amplitude plotted from the simplified expressions in Table 1. These plots show that the optimum modulation amplitudes for the four lowest even harmonics of the Fourier components $(n=2,4,6,8)$ are 2.20, 4.12, 6.08, and 8.06. The signal strengths are $-0.343,0.180,-0.122$, and 0.0915 , respectively.

\section{Conclusions}

The expressions derived here, which are significantly easier to work with than the general complex expression, make also investigations of multi-peak peak structures more manageable, which is of importance when atoms with either hyperfine structures or non-negligible isotope shifts are studied by WM techniques.

\section{References}

[1] Axner, P. Kluczynski, and A. M. Lindberg, "A general noncomplex analytical expression for the nth Fourier component of a wavelength-modulated Lorentzian lineshape function,'J. Quant. Spectrosc. Radiat. Transfer 68, 299-317 (2001).

[2] T. Svensson, M. Andersson, L. Rippe, S. Svanberg, S.Andersson-Engels, J. Johansson, and S. Folestad, "VCSELbased oxygen spectroscopy for structural analysis of pharmaceutical solids," Appl. Phys. B 90, 345-354 (2008).

[3] M. Lewander, Z. Guan, K. Svanberg, and S. Svanberg, "Clinical system for non-invasive in situ monitoring of gases in the human paranasal sinuses," Opt. Express 17, 10849-10863(2009).

[4] L. Mei, S. Svanberg, and G. Somesfalean, "Combined optical porosimetry and gas absorption spectroscopy in gas-filled porous media using diode-laser-based frequency domain photon migration,” Opt. Express 20, 16942-16954 (2012).

[5] L. Mei, G. Somesfalean, and S. Svanberg, "Light propagation in porous ceramics: porosity and optical property studies using tunable diode laser spectroscopy,” Appl. Phys. A 114,393-400 (2014).

[6] S. Schilt, L. Thevenaz, and P. Robert, "Wavelength modulation spectroscopy: combined frequency and intensity laser modulation,”Appl. Opt. 42, 6728-6738 (2003).

[7] Arndt R. Analytical line shapes for Lorentzian signals broadened by modulation. J Appl Phys 1965;36:2522-2524.

[8] Kluczynski P, Axner O. Theoretical description based upon Fourier analysis of wavelength-modulation spectrometry in terms of analytical and background signals. Appl Opt 1999、38: 5803-5815. 